\title{
Further spectral and chromatographic studies of ambergris
}

\author{
Steven J. Rowland ${ }^{* 1}$, Paul A. Sutton ${ }^{1}$, Simon T. Belt ${ }^{1}$, Vera Fitzsimmons-Thoss ${ }^{2}$ and Alan G. \\ Scarlett ${ }^{3}$
}

1. Petroleum and Environmental Geochemistry Group, Biogeochemistry Research Centre, University of Plymouth, Drake Circus, Plymouth, PL4 8AA, UK.

2. School of Chemistry, Bangor University, Bangor, Gwynedd, Wales, LL57 2UW, UK.

3. The Institute for Geoscience Research, Department of Chemistry, Curtin University, Perth, Western Australia.

*Corresponding Author:

Phone: $+44(0) 1752584557$

Fax: $\quad+44(0) 1752584710$

E-mail: srowland@plym.ac.uk

\footnotetext{
As per Taylor \& Francis Instructions to Authors

(http://www.tandfonline.com/action/authorSubmission?show=instructions\&journalCode=gnpl20\#Checklist, accessed $25^{\text {th }}$ October 2017), we include here all authors' full names, affiliations, postal addresses, telephone numbers and email addresses on the title

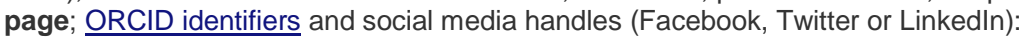

Steven John Rowland, Petroleum and Environmental Geochemistry Group, Biogeochemistry Research Centre, University of Plymouth, Drake Circus, Plymouth, PL4 8AA, UK; +44 (0)1752 584557, srowland@plymouth.ac.uk; ORCID 0000-0003-4980-0618.

Paul Andrew Sutton, Petroleum and Environmental Geochemistry Group, Biogeochemistry Research Centre, University of Plymouth, Drake Circus, Plymouth, PL4 8AA, UK; +44 (0)1752 584553; pasutton@plymouth.ac.uk; ORCID 0000-0003-0568-5478.

Simon Timothy Belt, Petroleum and Environmental Geochemistry Group, Biogeochemistry Research Centre, University of Plymouth, Drake Circus, Plymouth, PL4 8AA, UK; +44 (0)1752 5845, sbelt@plymouth.ac.uk; ORCID 0000-0002-1570-2924

Vera Fitzsimmons-Thoss, School of Chemistry, Bangor University, Bangor, Gwynedd, Wales, LL57 2UW, UK; +44 (0)1248 382516 . vera.thoss@bangor.ac.uk; ORCID 0000-0003-0915-5108.

Alan George Scarlett, The Institute for Geoscience Research, Department of Chemistry, Curtin University, Perth, Western Australia; +61 89266 3819, alan.scarlett@curtin.edu.au; ORCID 0000-0002-9559-2154
} 
doi: $\underline{10.1080 / 14786419.2018 .1428599}$

\section{Abstract}

Jetsam ambergris, found washed ashore on beaches, is an environmentally-modified form of a natural product of Sperm whales which sometimes develops a pleasant odor. Odorous samples have proved valuable in perfumery. Identification of jetsam ambergris by analysis of organicsoluble extracts by Fourier transform infra-red spectroscopy (FTIR) and of derivatised samples by gas chromatography-mass spectrometry (GC-MS) has already been shown. Here we describe a different method, in which characteristic alkenic protons and carbon atoms of the major constituent ambrein, were identified in whole extracts using nuclear magnetic resonance spectroscopy (NMR). The advantages of employing NMR spectroscopy included rapidity, reduced losses of volatiles compared to GC-MS and detection of non-GC amenable constituents. However, the identities and quantities of co-occurring individual components (e.g. steroids) could not easily be assigned in the unfractionated extracts by NMR spectroscopy, whereas they were by GC-MS, so an approach combining FTIR, GC-MS and NMR spectroscopic methods is advocated.

Keywords: Ambergris, NMR, GC-MS, ambrein, steroids, Sperm whale.

\section{Introduction}

Ambergris is a natural product which occurs only rarely as large coproliths in about $1 \%$ of Sperm whales (reviewed by Clarke 2006). Sometimes it is also washed up as jetsam on beaches, which can occur more or less worldwide (Kemp 2012). However, many jetsam samples, such as some of those reported in the media, are unverified: chemical analysis has revealed that few are in fact, ambergris (Sutton and Rowland 2018). Those jetsam samples which have been verified by analysis usually comprise mainly the terpenoid, ambrein (Figure 1) with low proportions of faecal steroids (Rowland and Sutton 2017). In contrast, Sperm whale ambergris (rather than jetsam) often contains much higher proportions of these steroids (Baynes-Cope 1962; Korzh and Strigina 1972; Rowland and Sutton 2017).

Depending on the conditions, environmental modification of jetsam ambergris may produce substances with distinctive odors. Odorous samples have been considered valuable in perfumery since ancient times (Lederer 1950; Freedman 2015). Indeed, ambergris is one of the more sought after raw materials used in perfumery. Its value to perfumers depends on the fixative properties of non-odorous, tricylic, ambrein and also on the composition of co-occurring 
very odorous, mono-, bi- and tricyclic volatile compounds (Serra 2013). These are formed naturally in trace amounts from ambrein by autoxidation or photo-oxidation; processes which may be simulated in the laboratory (Ohloff et al. 1972), or enhanced by 'ageing' in ethanolic solutions or tinctures (Mookherjee and Patel 1977; Awano et al. 2005).

However, in addition to its use as a fixative and precursor of odorants in perfumery, ambrein has also been reported to exhibit a number of biochemical and medical properties (Taha 1989, 1991, 1992; Taha et al. 1995, 1998; Raza et al. 2007).

As a result, the determination of ambrein content is important for verification of jetsam as ambergris (Rowland and Sutton 2017), whilst the evaluation of the value of ambergris samples for perfumery may also require characterization of the volatiles (Awano et al. 2005) and ideally, determination of the concentrations. Until recently, few analyses of whole unfractionated, lipidsoluble extracts of ambergris had been published. However, when a study of twelve extracts of ambergris from Sperm whales and jetsam was made by Fourier transform infra-red spectroscopy (FTIR) and, after derivatisation, by gas chromatography-mass spectrometry (GCMS) (Rowland and Sutton 2017), it was found that ambrein comprised between 26 and 97\% of the GC-MS detectable constituents in the soluble fractions. The other major constituents, which were detectable by GC-MS in some of the ambergris samples excised from Sperm whales, were the aforementioned faecal steroids (Rowland and Sutton 2017).

Since the latter GC-MS procedure required a derivatisation step prior to analysis (Governo et al. 1977), we sought to investigate additional, complementary analytical methods, which like FTIR, were rapid, but which were more specific than FTIR and which avoided the need for the derivatisation step used in the GC-MS method. ${ }^{1} \mathrm{H}$ NMR spectroscopic methods have been used occasionally to study chromatographic fractions of ambrein isolated from ambergris (Moniz and Hammond 1996), although neither ${ }^{1} \mathrm{H}$ nor ${ }^{13} \mathrm{C}$ NMR spectra of whole extracts have been reported, to our knowledge.

Therefore, we sought to investigate the characteristics of the ${ }^{1} \mathrm{H}$ and ${ }^{13} \mathrm{C}$ NMR spectra of whole extracts of two ambergris samples which were shown previously (Rowland and Sutton 2017) to be representative of the range of 'purities' encountered for ambergris. One was a jetsam sample determined previously to comprise relatively pure ambrein by GC-MS/derivatisation and one, excised from a Sperm whale, was shown to contain high proportions of co-occurring constituents, mainly a range of faecal steroids (Rowland and Sutton 2017). To add further to the very sparse database for the composition of ambergris from Sperm whales (Lederer et al. 1946; 
Please note: This is an accepted manuscript pre-publication. Readers are advised to consult the final, published version to avoid confusion:

doi: $\underline{10.1080 / 14786419.2018 .1428599}$

Lederer 1950; Baynes-Cope 1964; Rowland and Sutton 2017), GC-MS and FTIR data were also obtained for extracts of a further seven archived museum samples.

\section{Results and discussion}

${ }^{1} \mathrm{H}$ NMR spectroscopy

The proton NMR spectrum of a whole dichloromethane extract (100\% of the mass) of a silvercoloured sample of jetsam ambergris collected from a beach in New Zealand (Rowland and Sutton 2017), re-dissolved in deuterated chloroform, is shown in Figure 1a. Previously this extract was shown to comprise mainly ambrein $\left(\mathrm{C}_{30} \mathrm{H}_{52} \mathrm{O}\right)$ by $\mathrm{GC}-\mathrm{MS}$ of the trimethylsilyl ethers (Rowland and Sutton 2017).

Resonances at 4 to about 5 ppm (Figure 1a) in the ${ }^{1} \mathrm{H}$ NMR spectrum were assigned to alkenic protons by comparison with published spectra for synthetic ambrein (Oritani et al. 1970; Mori and Tamura 1990; Moniz and Hammond 1996) and enzymatically-produced ambrein (Ueda et al. 2013). Thus, using the original numbering system of Mori and Tamura (1990) for consistency, (as did Moniz and Hammond, 1996), the two broad singlets occurring at 4.5 and $4.7 \mathrm{ppm}$ were assigned to the two methylenic protons $\left(6 "=\mathrm{CH}_{2}\right)$. Similarly, the triplet at about 5.2 ppm was assigned to the vinylic proton at 3' (Figure 1a). Each of these resonances integrated quite closely to one proton, as expected (Figure 1a). These characteristic resonances allow the presence of ambrein (and identity of the origin of the sample as ambergris) to be assigned with reasonable certainty in this relatively pure extract. In addition, the resonances due to the methyl groups 4' and 2 were also assigned (Figure 1a). However, the presence of very minor unknown constituents, possibly too volatile for, or not amenable to, detection by GC-MS, was indicated by the other, minor, resonances in the region between 0.5 and $2.2 \mathrm{ppm}$. These underlay the characteristic resonances of pure ambrein (cf Mori and Tamura 1990; Moniz and Hammond 1996; Ueda et al. 2013).

In contrast, the ${ }^{1} \mathrm{H}$ NMR spectrum of the extract from a Sperm whale harvested in 1947 in the Southern Ocean (Clarke 2006) indicated a sample of much lower 'purity' (Figure 1c), with integration of the high field part of the spectrum ( 0-2 ppm) suggesting many other protons. Indeed, this sample seems to be typical of some ambergris samples obtained directly from whales (cf Figures S3, S4). This sample contained not only ambrein, but also large proportions of several faecal steroids, including epicoprostanol and coprostanone, as shown by GC-MS methods (Rowland and Sutton 2017). Even so, the characteristic resonances due to the alkenic 
doi: $\underline{10.1080 / 14786419.2018 .1428599}$

protons of ambrein were still recognised in the ${ }^{1} \mathrm{H}$ NMR spectrum (Figure 1c), as described above for the jetsam sample (Figure 1a). Thus, the ${ }^{1} \mathrm{H}$ NMR method was rapid (minutes of data acquisition), avoided the need for a derivatisation step and allowed detection of components which may not have been amenable to GC-MS. However, drawbacks with ${ }^{1} \mathrm{H}$ NMR spectroscopy included the need for relatively large samples (milligrams), the uncertainty of assignments in those cases where components other than ambrein were significantly abundant, and the difficulties in identifying and quantifying such components.

\section{${ }^{13} \mathrm{C}$ NMR spectroscopy}

The ${ }^{13} \mathrm{C}$ NMR spectrum of the whole dichloromethane extract of the silver-coloured jetsam ambergris collected from New Zealand, re-dissolved in deuterated chloroform, is shown in Figure 1b. Resonances at $\sim 100-150$ ppm were assigned to alkenic carbon atoms by comparison with a published spectrum for enzymatically-produced ambrein (Ueda et al. 2013). The resonances occurring in this region were each assigned to C6", 4', 3' and the methylenic $\mathrm{C} 6 "=\mathrm{CH}_{2}$. In addition, the resonance due to $\mathrm{C} 2$ was also assigned (Figure 1b). These features were distinctive and, with the ${ }^{1} \mathrm{H}$ NMR data above, again allowed the presence of ambrein to be confirmed. However, the presence of the steroids identified by GC-MS (Rowland and Sutton 2017) was also indicated by analysis of the signals in the 0 to $\sim 60 \mathrm{ppm}$ region of the spectrum which exceeded the 25 carbons required for the spectrum of pure ambrein (cf Ueda et al. 2013), albeit these additional signals were relatively minor in intensity (Figure $1 \mathrm{~b}$ ).

In comparison to the spectrum of the jetsam ambergris referred to above (Figure 1b), the spectrum (Figure 1d) of the extract from the Sperm whale was less well defined, as expected from the ${ }^{1} \mathrm{H}$ NMR spectrum. The characteristic resonances due to the diagnostic alkenic carbon atoms of ambrein could still be recognised, as described above for the jetsam sample (Figure $1 b)$, but the number of signals arising from carbon atoms of components other than ambrein, was much greater and more pronounced (Figure 1d).

\section{GC-MS}

Given the rather sparse current database for ambergris composition from whales (Lederer 1950; Baynes-Cope 1964; Rowland and Sutton 2017), we also obtained GC-MS and FTIR data of a further seven archived Sperm whale ambergris samples. Extraction and dissolution into dichloromethane, revealed that $84 \%$ to $96 \%$ of the whole masses of samples Albany 1-7 collected from Sperm whales harvested at the whaling station in Albany, Western Australia during the period estimated 1955 to 1978 (likely mostly towards the latter date), were 
doi: $\underline{10.1080 / 14786419.2018 .1428599}$

dichloromethane-soluble (Table S1). The insoluble material was not examined further. Each sample contained five main components and several trace components (A1-D, Figure S4). As expected, a distinctive component was ambrein (Figure S4, peak D), which had been converted efficiently to the TMS ether in the derivatised samples (Figure S4), as shown previously (Rowland and Sutton 2017). Minor amounts of other components, including ambroxan and ambreinolide, were identified in some samples by GC-MS (Figures S3 and S4). The proportions of steroids were also high (Figure S4). Thus it seems that many samples obtained directly from whales have higher proportions of faecal steroids relative to ambrein, than jetsam samples; though not all (Rowland and Sutton, 2017 and herein). The ratio of epicoprostanol to ambrein has been used previously as a measure of ambergris 'quality' (Baynes-Cope 1962; Korzh and Strigina 1972), but for perfumery, consideration of the (usually $<0.3 \%$ ) volatiles contents of mono, bi- and tricyclic oxidation products of ambrein, is also important (Serra 2013) Although ambergris tinctures have been examined, routine methods for simultaneous, reproducible, quantitative assay of volatiles, such as ambrox, ambrinol and ionones, in whole ambergris extracts, to our knowledge have yet to be published (Mookherjee and Patel 1977; Ohloff et al. 1977; Ohloff 1982; Awano et al. 2005).

\section{Conclusion}

The advantages of NMR spectroscopy for assignment of jetsam ambergris included the rapidity of the method and the lack of a need for derivatisation procedures. NMR spectral analysis also provided the capability of quickly ruling out jetsam samples that did not contain ambrein (i.e. which were not ambergris). However, co-occurring steroids could not be assigned or so easily quantified in the whole, unfractionated extracts, by NMR. An approach combining FTIR, GCMS and NMR methods is therefore advocated. For perfumery, simultaneous quantitative determination of volatile components may also be required. The latter will be the subject of future studies.

Acknowledgements We are grateful to Katelyn Weinert, archivist of the Albany Whaling Museum, Western Australia for supplying samples and information (to AGS) for whale ambergris 'Albany 1-7', originally collected 1955-1978. We would like to thank Denizcan Koseoglu (University of Plymouth) for obtaining and translating from Russian to English, a copy of Korzh and Strigina (1972) and Tony Wells and Jo Smith (Ambergris Connect Ltd) for a photograph of an ambergris sample found by G. McPhail, used in the graphical abstract. 
Please note: This is an accepted manuscript pre-publication. Readers are advised to consult the final, published version to avoid confusion:

doi: $\underline{10.1080 / 14786419.2018 .1428599}$

\section{References}

Awano K. Ishizaki S. Takazani O. Kitahara T. 2005. Analysis of ambergris tincture. Flav Frag J. 20:18-21.

Baynes-Cope AD. 1962. Analyses of samples of ambergris. Nature. 193:978-979.

Brito C. Jordao VL. Pierce GJ. 2016. Ambergris as an overlooked historical marine resource: its biology and role as a global economic commodity. JMBAUK. 96:585-596.

Clarke R. 2006. The origin of ambergris. LAJAM. 5:7-21.

Freedman P. 2015. Health, wellness and allure of spices in the Middle Ages. J Ethnopharm. 167:47-53.

Governo TF. Alessandro RT. Pragger MJ. 1977. Gas-liquid chromatographic-mass spectrometric detection and identification of ambergris. J AOAC Int. 60:160-164.

Kemp C. 2012. Floating gold : a natural (and unnatural) history of ambergris. University of Chicago Press. 232 pp.

Korzh LN. Strigina LI. 1972. Chemical composition of different varieties of ambergris. MasloZhirovaya Promyshlennost 10:25-26. (In Russian).

Lederer E. 1950. Odeurs et parfums des animaux. Progess in the chemistry of organic natural products 6 . (Zechmeister L, ed), 87-153, Springer-Verlag. Vienna.

Lederer E. Marx F. Mercier D. Perot G. 1946. Sur les constituants de l'ambre gris II. Ambreine et coprostanone. Helv Chim Acta. 29:1354-1365.

Mookherjee B. Patel R. 1977. Isolation and identification of volatile constituents of tincture ambergris. 7th International Congress of Essential Oils. 136:479-482.

Moniz GA. Hammond GB. 1996. Identification of ambergris from the New Bedford whaling museum by nuclear magnetic resonance spectroscopy. J AOAC Int. 79:423-425.

Ohloff G. 1982. The fragrance of ambergris. Chapter 15. Fragrance chemistry. Academic Press. 535-573. 
Please note: This is an accepted manuscript pre-publication. Readers are advised to consult the final, published version to avoid confusion:

doi: $\underline{10.1080 / 14786419.2018 .1428599}$

Ohloff G. Schulte-Elte KH Müller BL. 1977. Formation of ambergris odorants from ambrein under simulated natural conditions. Helv Chim Acta 60:2763-2766

Mori K. Tamura H. 1990. Triterpenoid total synthesis I. Synthesis of ambrein and Ambrox®. Liebigs Ann. Chem. 361-368.

Oritani T. Yamashita K. Matsui M. 1970. Chemical studies of ambergris. Part IV. Configuration of ambrein. Agri Biol Chem. 34:1244-1248.

Taha SA. 1989. Chemical investigation of the internal secretion of the Sperm blue whale. Pakistan J Pharm Sci. 2:105-110.

Taha SA. 1991. Effect of ambrein on blood glucose levels of rats. J Ethnopharm. 35:145-148.

Taha SA. 1992. Studies on the mode of action of ambrein as a new antinociceptive compound. Japan J Pharmacol. 60:67-71.

Taha SA. Islam MW. Ageel AM. 1995. Effect of ambrein, a major constituent of ambergris, on masculine sexual behavior in rats. Archives Internat Pharmacodynamie Thérapie. 60:19-26.

Taha SA. Raza M. El-Khawad IE. 1998. Effect of ambrein on smooth muscle responses to various agonists. J Ethnopharm. 60:19-26.

Raza M. Alorainy MS. Alghasham A. 2007. Evaluation of ambrein and epicoprostanol for their antioxidant properties: protection against adriamycin-induced free radical toxicity. Food Chem Toxicol. 45:1614-1619.

Rowland SJ. Sutton PA. 2017. Chromatographic and spectral studies of jetsam and archived ambergris. Nat Prod Res. 15:1752-1757.

Schaefer B. 2014. Natural products in the chemical industry. Springer. 831 pp.

Serra S. 2013. An expedient preparation of enantio-enriched ambergris odorants starting from commercial ionone alpha. Flavour Frag J. 28:46-52. 
Please note: This is an accepted manuscript pre-publication. Readers are advised to consult the final, published version to avoid confusion:

doi: $\underline{10.1080 / 14786419.2018 .1428599}$

Sutton PA Rowland SJ. 2018. Analysis of miscellaneous non-ambergris organic jetsam beach deposits. Earth ArXiv Pre-print doi: 10.17605/OSF.IO/9CDYU . Available at https://eartharxiv.org.

Ueda D. Hoshino T. Sato T. 2013. Cyclisation of squalene from both termini: identification of an onoceroid synthase and enzymatic synthesis of ambrein. J Am Chem Soc. 135:18335-18338.

Figure 1. NMR spectra of dichloromethane extracts of ambergris, redissolved in deuterated chloroform from a jetsam sample from New Zealand $(a, b)$ and from a Sperm whale from the Southern Ocean (c, d). The structure of the major component, ambrein $\mathrm{C}_{30} \mathrm{H}_{52} \mathrm{O}$, is also shown. The numbering system is that employed by Mori and Tamura 1990. Numbers in black on the spectra correspond to the integrations $\left({ }^{1} \mathrm{H}\right)$ or number of identifiable resonances above the noise $\left({ }^{13} \mathrm{C}\right)$. Numbers in red on the spectra indicate the positions of $\mathrm{H}$ and $\mathrm{C}$ atoms in ${ }^{1} \mathrm{H}$ and ${ }^{13} \mathrm{C}$ spectra respectively. 
Please note: This is an accepted manuscript pre-publication. Readers are advised to consult the final, published version to avoid confusion:

\section{dol: $10.1080 / 14786419.2018 .1428599$}
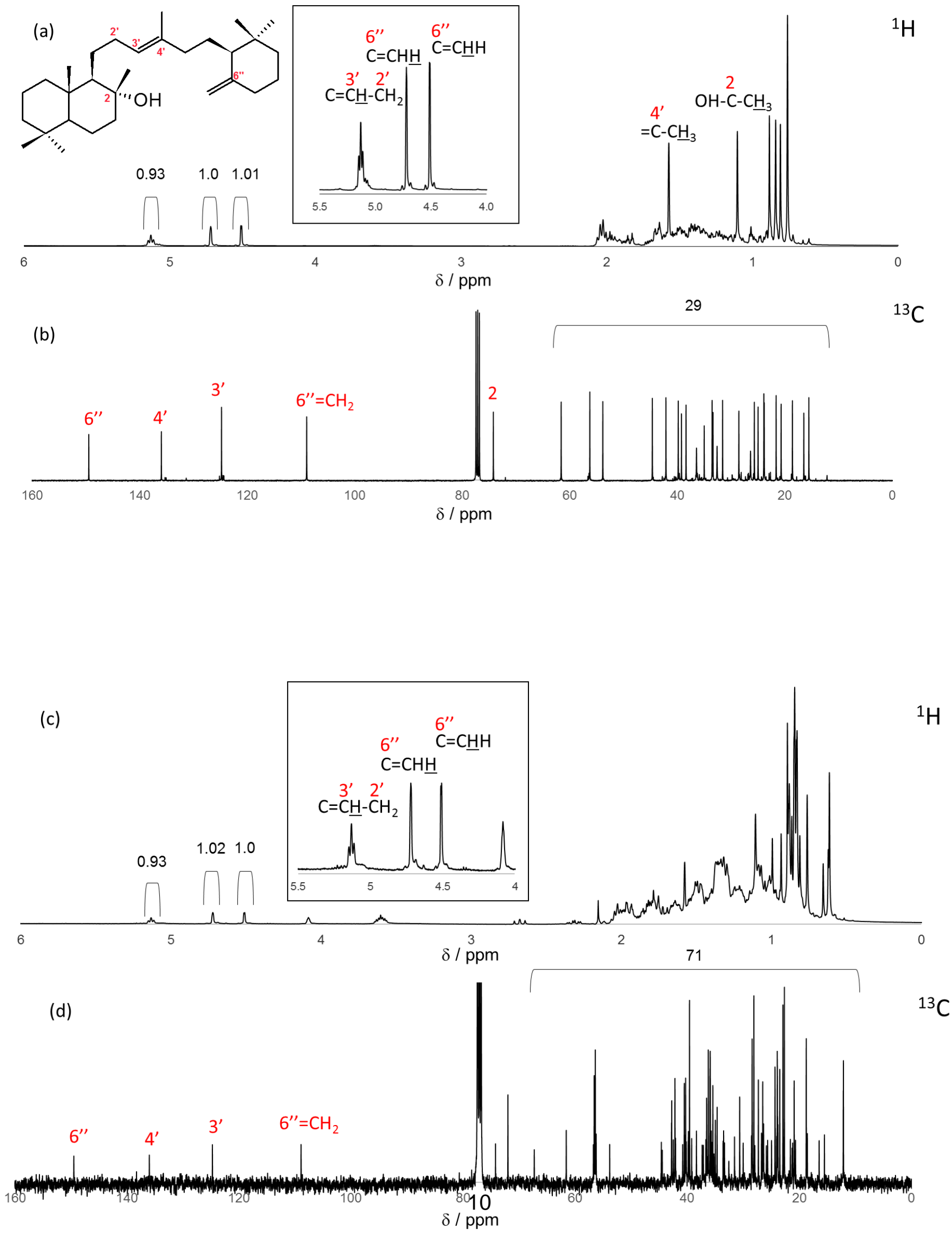


\section{Further spectral and chromatographic studies of ambergris}

Steven J. Rowland ${ }^{* 1}$, Paul A. Sutton ${ }^{1}$, Simon T. Belt ${ }^{1}$, Vera Fitzsimmons-Thoss ${ }^{2}$ and Alan G. Scarlett ${ }^{3}$

1. Petroleum and Environmental Geochemistry Group, Biogeochemistry Research Centre, University of Plymouth, Drake Circus, Plymouth, PL4 8AA, UK.

2. School of Chemistry, Bangor University, Bangor, Gwynedd, Wales, LL57 2UW, UK.

3. The Institute for Geoscience Research, Department of Chemistry, Curtin University, Perth, Western Australia.

*Corresponding Author:

Phone: +44 (0)1752 584557

Fax: $\quad+44(0) 1752584710$

E-mail: srowland@plym.ac.uk 
Abstract

Jetsam ambergris, found washed ashore on beaches, is an environmentally-modified form of a natural product of Sperm whales which sometimes develops a pleasant odor. Odorous samples have proved valuable in perfumery. Identification of jetsam ambergris by analysis of organicsoluble extracts by Fourier transform infra-red spectroscopy (FTIR) and of derivatised samples by gas chromatography-mass spectrometry (GC-MS) has already been shown. Here we describe a different method, in which characteristic alkenic protons and carbon atoms of the major constituent ambrein, were identified in whole extracts using nuclear magnetic resonance spectroscopy (NMR). The advantages of employing NMR included rapidity, reduced losses of volatiles compared to GC-MS and detection of non-GC amenable constituents. However, the identities and quantities of co-occurring individual components (e.g. steroids) could not easily be assigned in the unfractionated extracts by NMR, whereas they were by GC-MS, so an approach combining FTIR, GC-MS and NMR methods is advocated.

Keywords: Ambergris, NMR, GC-MS, ambrein, steroids, Sperm whale.

\section{Experimental}

\section{Materials}

A boulder of ambergris weighing $155 \mathrm{~kg}$ taken from a $16 \mathrm{~m}$ male Sperm whale on board

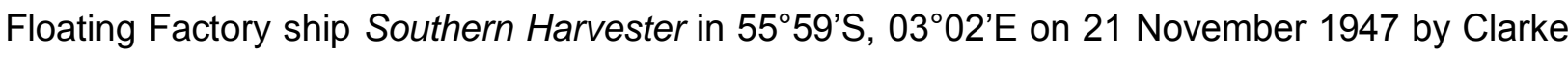
[Clarke 2006 shows a photograph taken at the time], portions of which are archived by the Natural History Museum, London, was sub-sampled by one of the present authors (SJR) on 14 June 2016. Small pieces were taken from an odorous black fragment with a smell similar to that of synthetic Ambrox ${ }^{\circledR}$, covered in white crystals (Rowland and Sutton 2017, Sample 1). The sample was $25 \%$ soluble in dichloromethane and $26 \%$ of the GC-MS detectable material was assigned as ambrein (as TMS ether; Rowland and Sutton 2017). A sample of silvery, slightly odorous confirmed jetsam ambergris (Rowland and Sutton 2017, Sample 11) was provided from a beach from an undisclosed location in New Zealand. The sample was completely soluble in dichloromethane and $97 \%$ of the GC-MS detectable material was assigned as ambrein (as 
Please note: This is an accepted manuscript pre-publication. Readers are advised to consult the final, published version to avoid confusion:

doi: $10.1080 / 14786419.2018 .1428599$

the TMS ether; Rowland and Sutton 2017). Seven ambergris samples (Albany 1-7) were donated by Albany's Historic Whaling Museum (Discovery Bay), Albany, Western Australia, Australia and supplied (to AGS) by Katelyn Weinert, Archivist, on 17th Jan 2017. These samples (Figure S1) had originally been taken from harvested Sperm Whales from the Southern Ocean during 1955-1978 and archived by the museum. An authentic sample of ambrein was obtained from Ueda et al. (2013) and samples of ambrinol and ambroxan were supplied by a perfumery company. N,O-bis(trimethylsilyl)acetamide + trimethylchlorosilane $+\mathrm{N}$ trimethylsilylimidazole; BSA+TMCS+TMSI (3:2:3; 'Sylon BTZ') reagent was purchased from Sigma-Aldrich (Poole, UK).

\begin{tabular}{|c|l|l|c|}
\hline Sample & \multicolumn{1}{|c|}{ Aroma } & \multicolumn{1}{|c|}{$\begin{array}{c}\text { Appearance \& } \\
\text { Hardness }\end{array}$} & $\begin{array}{c}\text { \% } \\
\text { dichloromethane } \\
\text {-extractable }\end{array}$ \\
\hline Albany 1 & slight & $\begin{array}{l}\text { Brown mottled } \\
\text { solid. Pliable. }\end{array}$ & 94 \\
\hline Albany 2 & v.slight & $\begin{array}{l}\text { Brown mottled } \\
\text { solid. Brittle. }\end{array}$ & 96 \\
\hline Albany 3 & v.slight & $\begin{array}{l}\text { Brown mottled } \\
\text { solid. Brittle. }\end{array}$ & 84 \\
\hline Albany 4 & v.slight & $\begin{array}{l}\text { Brown mottled } \\
\text { solid. Brittle. }\end{array}$ & 92 \\
\hline Albany 5 & v.slight & $\begin{array}{l}\text { Brown mottled } \\
\text { solid. Hard to } \\
\text { break. }\end{array}$ & 95 \\
\hline Albany 6 & v.slight & $\begin{array}{l}\text { Brown mottled } \\
\text { solid. Brittle. }\end{array}$ & 90 \\
\hline Albany 7 & v.slight & $\begin{array}{l}\text { Brown mottled } \\
\text { solid. Brittle. }\end{array}$ & 84 \\
\hline
\end{tabular}

Table S1 Sample descriptions and \% dichloromethane-extractable material for Sperm whale ambergris collected Southern Ocean 1955-1978 and archived by Albany's Historic Whaling Museum (Discovery Bay), Albany, Western Australia, Australia. Received for study $17^{\text {th }}$ January 2017. 

consult the final, published version to avoid confusion:

doi: $\underline{10.1080 / 14786419.2018 .1428599}$

Figure S1. Photograph of ambergris samples Albany 1-7. Photo: AGS.

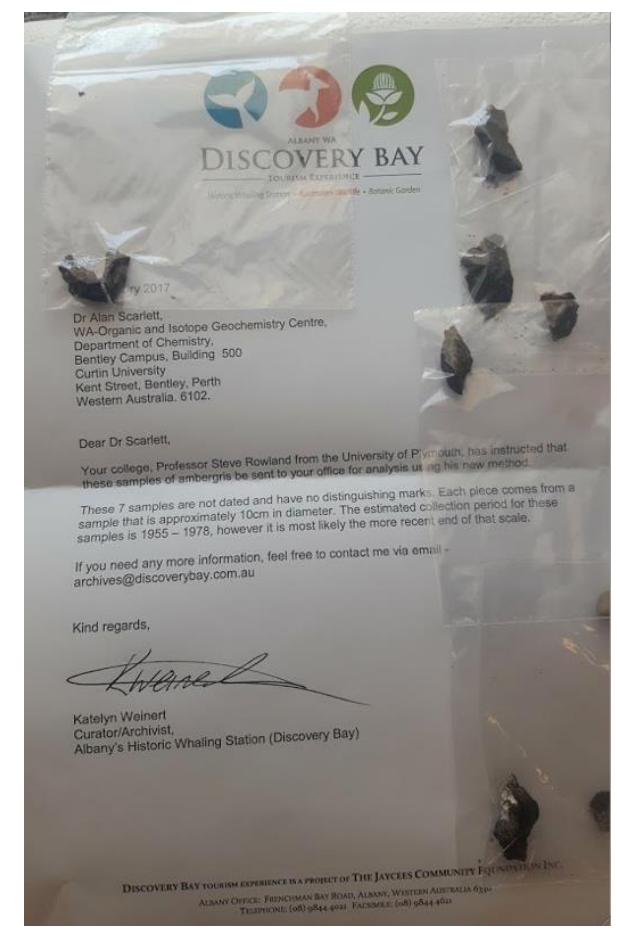

\section{Methods}

Core samples were taken from specimens of ambergris using an acetone rinsed scalpel blade and spatula and digested by sonication ( $2 \times 5 \mathrm{~min})$ in dichloromethane (Table S1). Samples for Fourier Transform infrared spectroscopy (FTIR) analysis were prepared at a nominal concentration of $10 \mathrm{mg} \mathrm{mL}^{-1}$. Aliquots of the digest were taken and diluted for direct analysis of the underivatised sample, or dried under a gentle stream of nitrogen $\left(40^{\circ} \mathrm{C}\right)$ and derivatised to their trimethylsilylated derivatives with BSA/TMCS/TMSI (3:2:3; 'Sylon BTZ'; $100 \mu \mathrm{L} ; 70^{\circ} \mathrm{C} ; 1 \mathrm{~h}$ ) and reconstitution in solvent, prior to analysis. Samples for NMR analysis were blown down to dryness in a gentle stream of nitrogen gas then re-dissolved in deuterated chloroform $\left(\mathrm{CDCl}_{3}\right)$. NMR spectroscopy was performed using a JEOL ECP-400 NMR Spectrometer with chemical shifts measured relative to $\mathrm{CDCl}_{3}\left({ }^{1} \mathrm{H}: 7.24 \mathrm{ppm} ;{ }^{13} \mathrm{C}: 77.0 \mathrm{ppm}\right)$. 
doi: $\underline{10.1080 / 14786419.2018 .1428599}$

FTIR was undertaken using a Bruker Alpha Platinum ATR (Bruker (UK) Ltd., Coventry, UK) instrument in transmittance mode (32 background and sample scans; resolution $4 \mathrm{~cm}^{-1}$ ) and data were collected from 4000 to $375 \mathrm{~cm}^{-1}$.

GC-MS was carried out using an Agilent GC-MSD (Agilent Technologies, Wilmington, DE, USA). This comprised a 7890A gas chromatograph fitted with a 7683B Series autosampler and a $5975 \mathrm{~A}$ quadrupole mass selective detector operated at $70 \mathrm{eV}$ ionisation voltage. The column was a HP-5MS fused silica capillary column (30 m x $0.25 \mathrm{~mm}$ i.d $\times 0.25 \mu \mathrm{m}$ film thickness). The carrier gas was helium at a constant flow of $1.0 \mathrm{~mL} \mathrm{~min}^{-1}$. A $1.0 \mu \mathrm{L}$ sample was injected into a $250{ }^{\circ} \mathrm{C}$ splitless injector. The oven temperature was programmed from 40 to $300{ }^{\circ} \mathrm{C}$ at $10^{\circ} \mathrm{C}$ $\mathrm{min}^{-1}$ and held for $10 \mathrm{~min}$ and the transfer line and source temperatures maintained at $280^{\circ} \mathrm{C}$ and $230^{\circ} \mathrm{C}$, respectively.

FTIR spectra of the samples were all broadly similar (Figure S2) and exhibited the following features: a broad transmittance at $3372 \mathrm{~cm}^{-1}$ was attributed to $\mathrm{H}$-bonded hydroxyl $\mathrm{O}-\mathrm{H}$ stretching. A weak transmittance at $\sim 3063 \mathrm{~cm}^{-1}$ was indicative of unsaturation and attributed to $\mathrm{C}-\mathrm{H}$ stretch in an alkene. Transmittances at 2925 and $2863 \mathrm{~cm}^{-1}$ were attributed to C-H stretching in methyl and methylene groups and those at 1457 and $1385 \mathrm{~cm}^{-1}$ to the corresponding bending vibrations. Weak transmittances at $1706 \mathrm{~cm}^{-1}$ were indicative of ketonic carbonyl groups ( $\mathrm{C}=\mathrm{O}$ stretch). Transmittances at $1644 \mathrm{~cm}^{-1}$ were attributed to $\mathrm{C}=\mathrm{C}$ stretch and at 935 and $887 \mathrm{~cm}^{-1}$ to $\mathrm{C}-\mathrm{H}$ out of plane bends in alkenes. The spectra were similar in several aspects to those of Rowland and Sutton (2017) and that published by Governo et al. (1977) for a sample of ambrein isolated from ambergris: the presence of the transmittance attributed to the keto group in the latter was not explained by the latter authors, but the other features are consistent with the known structure of ambrein and with the spectrum of synthetic ambrein. 
Please note: This is an accepted manuscript pre-publication. Readers are advised to consult the final, published version to avoid confusion:

doi: $\underline{10.1080 / 14786419.2018 .1428599}$

Figure S2. FTIR spectra of extracts of jetsam ambergris collected from a beach in New Zealand (Silver, reference; Rowland and Sutton 2017) and samples of whale ambergris (Albany 1-7) originally taken from harvested Sperm Whales from the Southern Ocean during 1955-1978. Wavenumbers $\left(\mathrm{cm}^{-1}\right)$ of diagnostic transmittance features of ambrein are indicated.

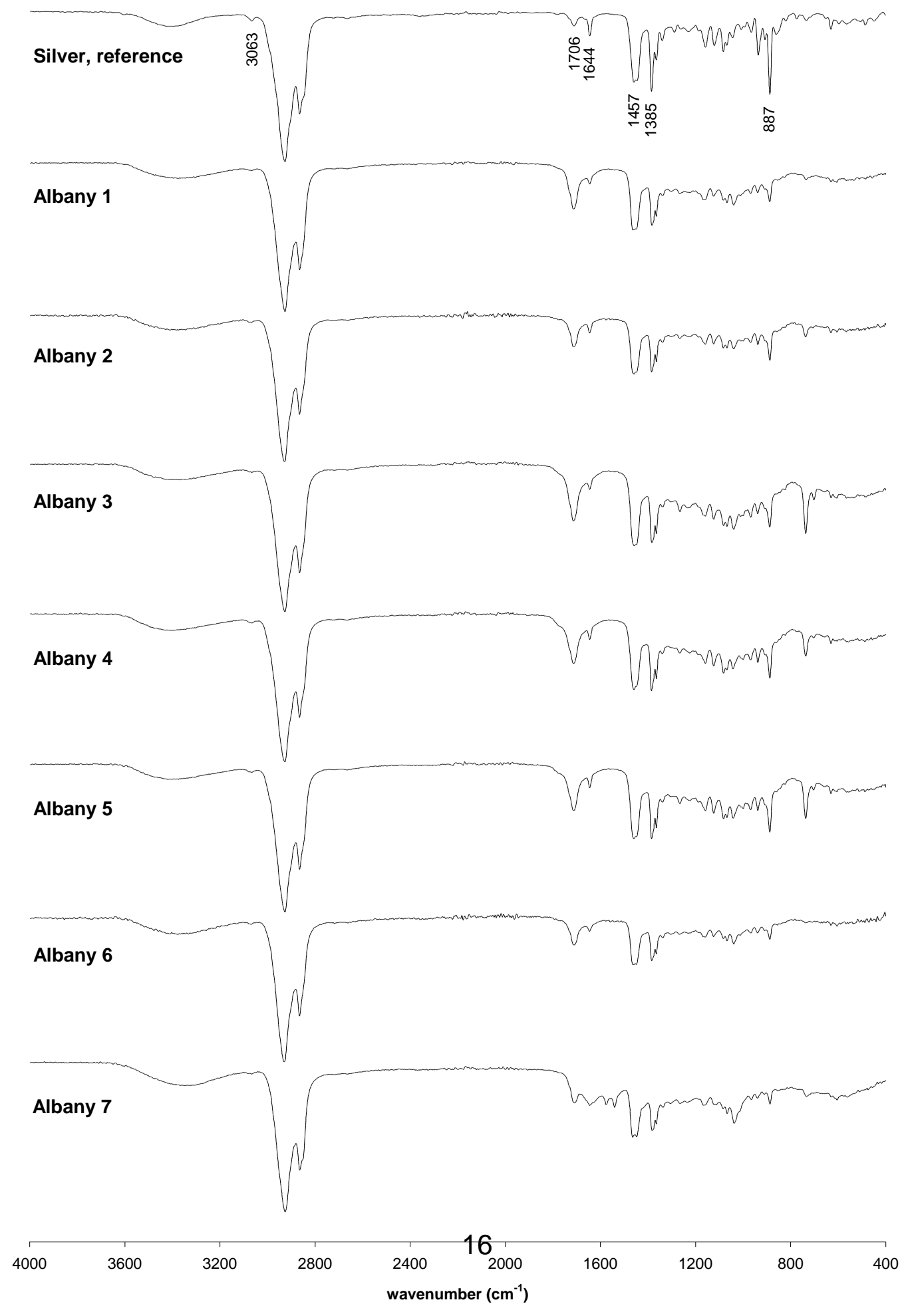


Please note: This is an accepted manuscript pre-publication. Readers are advised to consult the final, published version to avoid confusion:

doi: $\underline{10.1080 / 14786419.2018 .1428599}$

Figure S3. GC-MS total ion current chromatograms of a mixture of reference n-alkanes $\left(\mathrm{C}_{25-34}\right)$ and extracts of a non-derivatised sample of 'silver' jetsam ambergris from New Zealand (Sample 11, Rowland and Sutton 2017, 97\% ambrein) and of non-derivatised samples (Albany 1-7) of ambergris from Southern Ocean Sperm whales collected 1955-1978. Components E are three isomeric ambratrienes, artifacts resulting from in-injector dehydration of ambrein, identified by mass spectral interpretation and comparison with the data of Governo et al. (1977); component D comprises co-eluting epicoprostanol and ambrein. Minor early-eluting components (ca. 20-22 minutes retention time) include ambroxan and ambreinolide and other known and unknown components, likely of autooxidation of ambrein (Ohloff, 1982), identified herein by comparison of mass spectra with published spectra (e.g. Mori and Tamura 1990). 
Please note: This is an accepted manuscript pre-publication. Readers are advised to consult the final, published version to avoid confusion:

doi: $10.1080 / 14786419.2018 .1428599$

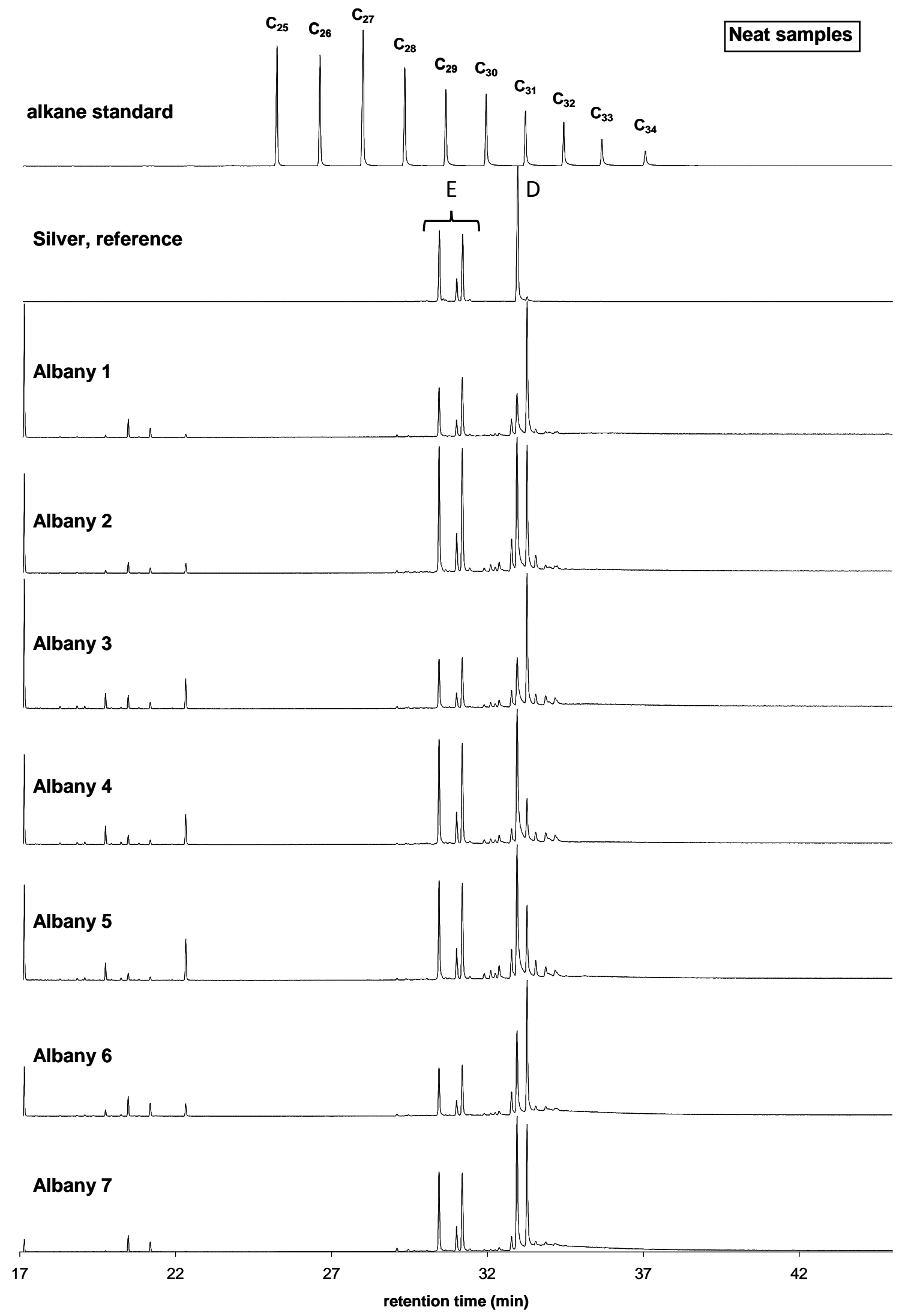


Please note: This is an accepted manuscript pre-publication. Readers are advised to consult the final, published version to avoid confusion:

doi: $\underline{10.1080 / 14786419.2018 .1428599}$

Figure S4. GC-MS total ion current chromatograms of a mixture of reference $n$-alkanes $\left(\mathrm{C}_{25-34}\right)$ and extracts of a derivatised sample of 'silver' jetsam ambergris from New Zealand (Sample 11, Rowland and Sutton 2017, 97\% ambrein) and of derivatised samples (Albany 1-7) of ambergris from Southern Ocean Sperm whales collected 1955-1978. Components A1, 2 are unknowns; component B is coprostanone; component C is epicoprostanol TMS ether and component $\mathrm{D}$ is ambrein TMS ether. Minor early-eluting components (ca. 20-25 minutes retention time) included ambroxan and ambreinolide, identified herein by comparison of mass spectra with published spectra (e.g. Mori and Tamura 1990). 
Please note: This is an accepted manuscript pre-publication. Readers are advised to consult the final, published version to avoid confusion:

doi: $\underline{10.1080 / 14786419.2018 .1428599}$

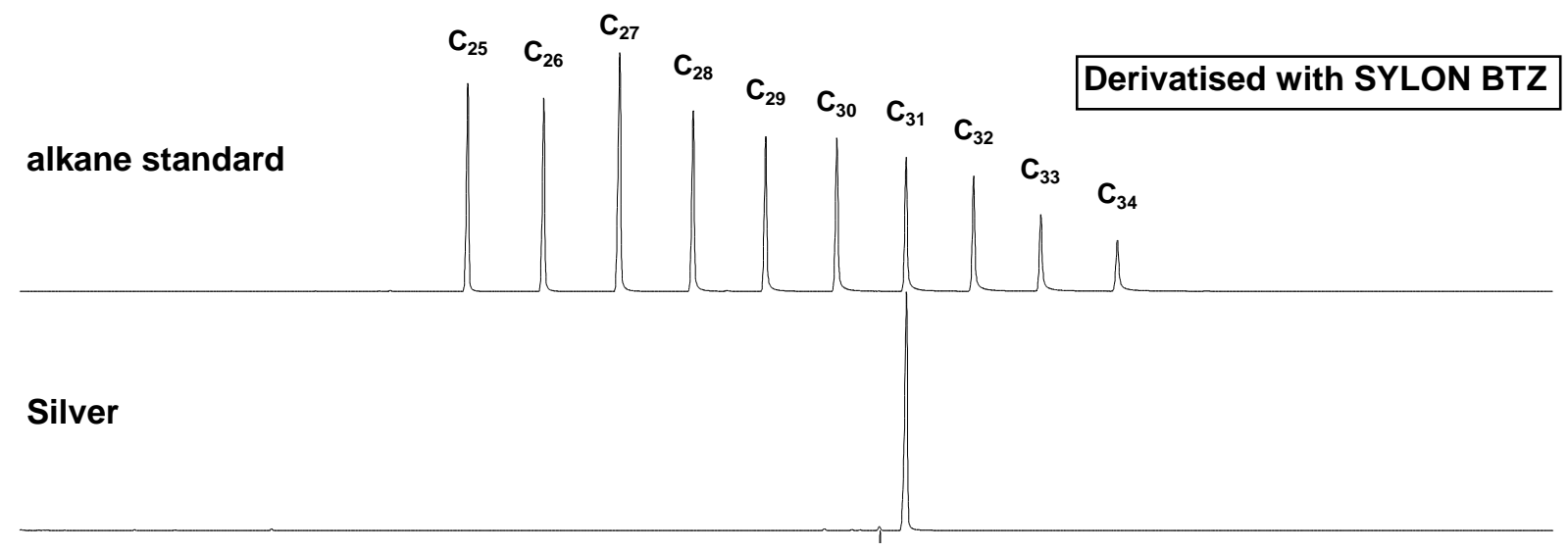

C D

Albany 1

$\mathrm{B}$

A1

Albany 2

Albany 3

Albany 4

Albany 5

Albany 6
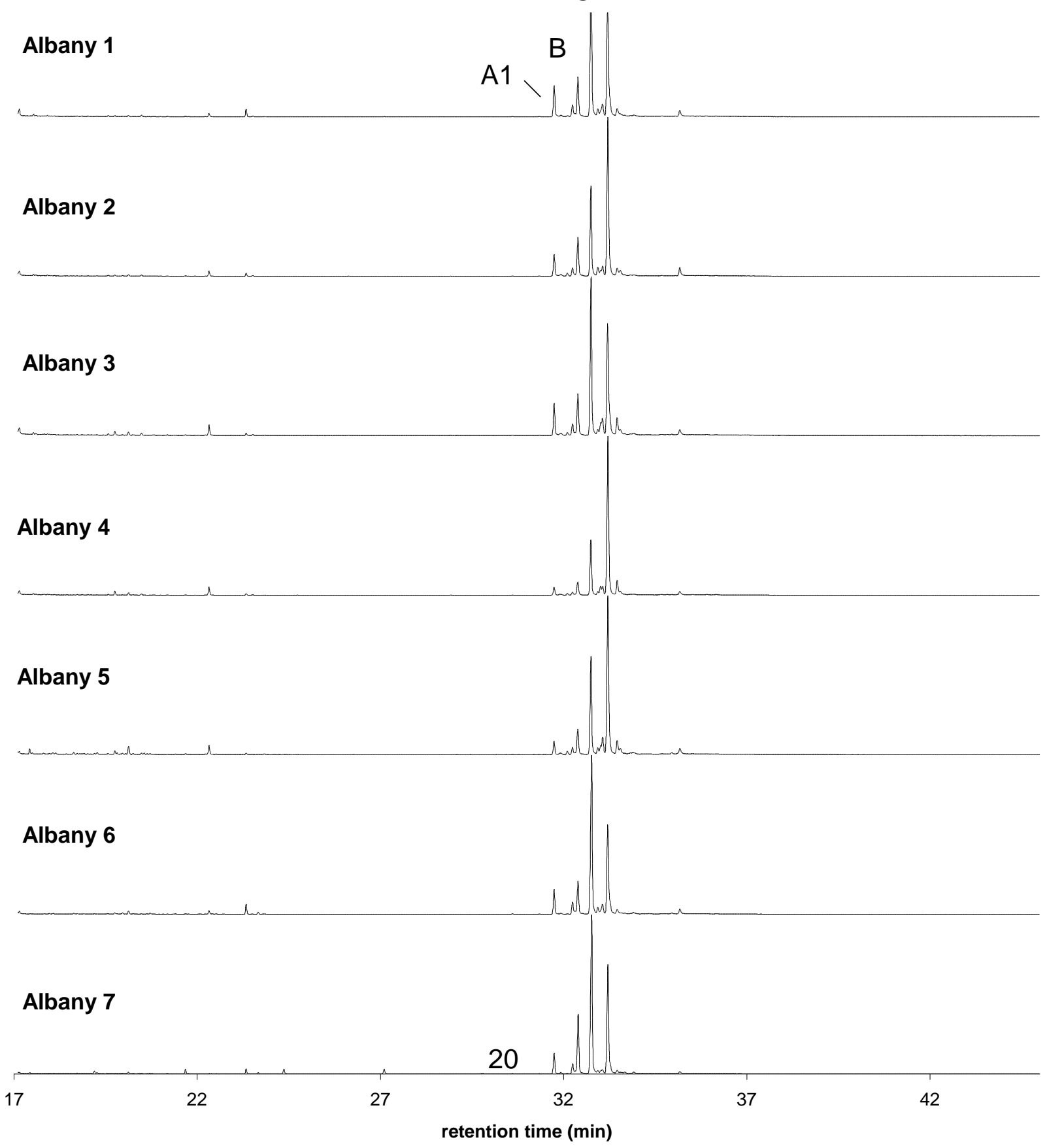